\title{
Exposure homogeneity of pharmaceutical materials in reverberation chamber
}

\author{
Sullivan Derenne ${ }^{1}$, François Horlin, Jean Michel Dricot and Philippe De Doncker ${ }^{2}$ \\ ${ }^{1}$ Université Libre de Bruxelles - OPERA Dpt. - Wireless Communications Group, Av. Franklin Roosevelt, 50 B-1050 \\ Brussels, Belgium.sderenne@ulb.ac.be ${ }^{1}$, pdedonck@ulb.ac.be ${ }^{2}$
}

\begin{abstract}
The growing interest of using RFID technology in the pharmaceutical domain leads to questions about the EMF exposure of drugs. In this sense, different types of exposure systems have been studied and in particular the exposure in reverberation chamber. In order to expose multiple drug samples simultaneously, this study evaluate the difference in exposure homogeneity between one or multiple samples exposition. Simulations give condition on both the size and the distance between the samples to reach homogeneity.
\end{abstract}

\section{Introduction}

The Radio Frequency Identification technology is used in lots of different applications for its advantages like the ability to trace and track some objects in a well-defined scenario from computer without human intervention. The interest of the auto-identification is now growing in the pharmaceutical domain for both the tracking and the security for counterfeit. Application in the pharmaceutical supply chain can be tracking of a unique drug or a box or an entire pallet. At certain logistic steps, the tags are interrogated by the RFID reader to download the data which are dependent on the capacities of the tag. The information collected by the RFID tag can be from as simple as the serial number or with more complex information like date, path or in more sensitive case with the addition of sensors, the history of temperature and humidity. The RFID technology can be used at different frequencies but this study focus on Ultra High Frequency (UHF) and more precisely on the $860 \mathrm{MHz}$ band although the methodology can be reproduced at all the UHF band.

The use of RFID systems in the pharmaceutical supply chain raises questions in terms of radiation impacts and especially for temperature sensitive pharmaceutical drugs. This study is part of the recent trend in evaluating the potential effects on pharmaceutical samples due to the radiation of RFID systems. The potential effects can be split into two categories, the thermal and non-thermal ones. Previous studies have also defined a methodology to evaluate the impact on both sides [1]. In addition to this distinction, it must be noticed that both the drug and the container can be affected by the radiation.

For irradiating samples, anechoic chamber is most often used as exposure system. In this study, we follow an other way and assume that it can be interesting to place the drug in a reverberation chamber. This can ensure high level field and isotropic irradiation of the sample. We have evaluated the possibility and the efficiency of placing more than one sample at once during an irradiation by simulating in two dimensions the reverberation chamber behavior.

\section{Context and scenario}

The methodology of irradiation chosen in this study is the exposition of the drug and its container in a reverberation chamber. The basic test consist in placing the sample in the middle of the test area of the reverberation chamber where this area provides isotropic and homogeneous statistic properties of the fields. In practice, all the preparation and the realization of the test take time and because of pharmaceutical constraints, we need to expose a lot of drips to ensure the reproducibility.

As a first step, this study consists in two dimensions simulations where we consider the drips as cylinder. Different scenario will be investigated, first in term of positioning the cylinders in the area. It will also include different size of drips (or cylinders) and different distance between the cylinders.

In order to evaluate the homogeneity of the exposure in the multi cylinders case, we will calculate the Specific Absorption Rate (SAR) in each cylinder and try to compare with the reference. The reference is a cylinder exposed alone in the test area. The evaluation of the homogeneity will be split into two steps. The first step consists in dividing each 
cylinder in cones and compare respectively each cone from a multi-drips case to the cone of reference. The second consists in dividing each cylinder in concentric rings and also for each ring compare to the reference. With these two steps we can evaluate the homogeneity in the penetration and directional points of view. Figure 1.a shows the unique cylinder used as reference, it is divided in cones and in concentric rings. Figure 1.b is an example of the rectangular disposition where each cylinder is divided into cones. Figure 1.c is a rectangular disposition where each cylinder is divided into concentric rings.

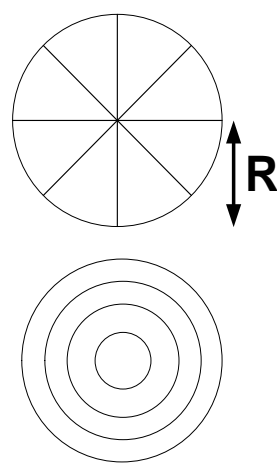

Figure 1.a Unique cylinder division

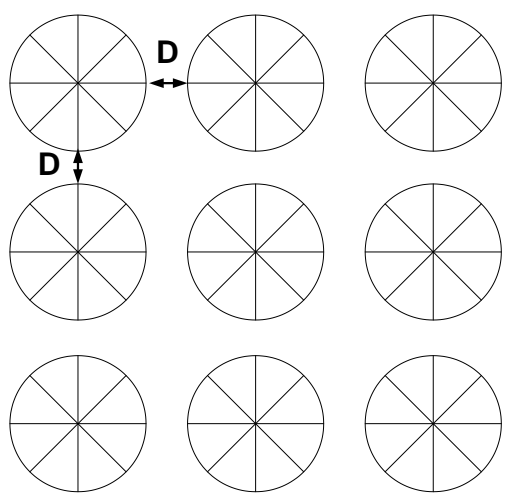

Figure 1.b Rectangular form and cone division

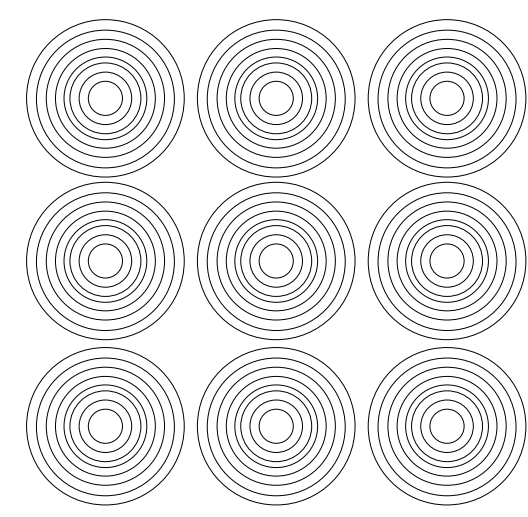

Figure 1.c Cross form and ring division

\section{Analytical method}

As a first step to simulate the exposition conditions in a reverberation chamber we consider a two dimensional scenario. The model of cylinder exposition is flexible in term of the number, the size and the disposition of the cylinders so we can simulate all the scenario necessary to this study. By assuming a plane wave incidence on the cylinder array, an iterative method is used to compute the electric field [2]. The scattered fields inside and outside every cylinder are computed at each iteration where for a plane wave till convergence is reached.

With the latter simulation we can obtain the electric field for one incidence direction separately but the goal is to approach the reverberation chamber conditions. To do this we need to calculate the total field for all MulitPath components (MPC) with random phase arriving isotropically on the cylinder array. The total field absorbed by the cylinders can be split into its random and deterministic parts:

$$
E_{t o t}=\sum_{l=1}^{N_{M P C s}} \varepsilon_{l}\left(\rho, \theta, \theta_{l}\right) \alpha_{l}
$$

Where $\varepsilon_{1}$ represents the normalized electric field (deterministic part) due to the 1-th MPC, $\rho$ and $\theta$ the cylindrical coordinates and $\alpha_{1}$ the complex amplitude having a random phase. We want to obtain the mean and the standard deviation of the total SAR $\mathrm{wB}_{\mathrm{B}}$. Under Uncorrelated Scattering (US) assumption the result given by [3]

$$
\left\langle S A R_{W B}\right\rangle=\frac{1}{m} \frac{P}{2 N_{M P C S}} \sum_{l=1}^{N_{M P C S}} \int_{V} \sigma\left|\varepsilon_{l}\left(\rho, \theta, \theta_{l}\right)\right|^{2} d V
$$

Where $\mathrm{P}$ is the power of the MPC and $\sigma$ the conductivity.

$$
\sigma_{S A R_{W B}}^{2}=\left\langle S A R_{W B}^{2}\right\rangle-\left\langle S A R_{W B}\right\rangle^{2}
$$

Becomes : 


$$
\sigma_{S A R_{W B}}=\frac{\sigma}{2 m} \frac{P}{N_{M P C S}}\left[\int_{V} \sum_{l \neq n}^{N_{M P C S}}\left|\varepsilon_{l}\left(\rho, \theta, \theta_{l}\right)\right|^{2}\left|\varepsilon_{n}\left(\rho, \theta, \theta_{n}\right)\right|^{2} d V\right]^{\frac{1}{2}}
$$

\section{Results}

Table 1 presents the different radii and distance between cylinders used in the simulations.

Table 1 : Radius and Distance for all the scenario

\begin{tabular}{|l|c|c|c|c|c|c|c|c|c|c|c|c|}
\hline Case & $\mathbf{1}$ & $\mathbf{2}$ & $\mathbf{3}$ & $\mathbf{4}$ & $\mathbf{5}$ & $\mathbf{6}$ & $\mathbf{7}$ & $\mathbf{8}$ & $\mathbf{9}$ & $\mathbf{1 0}$ & $\mathbf{1 1}$ & $\mathbf{1 2}$ \\
\hline Radius & $\lambda / 16$ & $\lambda / 16$ & $\lambda / 16$ & $\lambda / 16$ & $\lambda / 8$ & $\lambda / 8$ & $\lambda / 8$ & $\lambda / 8$ & $\lambda / 4$ & $\lambda / 4$ & $\lambda / 4$ & $\lambda / 4$ \\
\hline Distance & $\lambda / 8$ & $\lambda / 4$ & $\lambda / 2$ & $\lambda$ & $\lambda / 8$ & $\lambda / 4$ & $\lambda / 2$ & $\lambda$ & $\lambda / 8$ & $\lambda / 4$ & $\lambda / 2$ & $\lambda$ \\
\hline
\end{tabular}

The results of simulations about the standard deviation leads to a clear conclusion about the method. The difference between the standard deviations of the unique cylinder and one cylinder among the nine exposed simultaneously is negligible. So the method for one or for multiple cylinders gives results with the same deviation for the SAR.

Figure 2.a shows the mean (for all nine cylinders) spread for the cone division as a function of the distance between the cylinders for the three radii. Figure 2.b shows the mean spread for the concentric ring division. The spread is calculated by :

$$
S_{c}=\sqrt{\frac{\sum_{i=1}^{n}\left(S A R 9_{c, i}-S A R 1_{i}\right)^{2}}{\sum_{i=1}^{n} S A R 1_{i}{ }^{2}}}
$$

Where SAR9 $9_{c, i}$ represents the SAR for the i-th area (either for the cone or the ring division) of the c-th cylinders among the multiple cylinders and $S A R 1_{i}$ the $S A R$ for the $i$-th area of the reference cylinder.

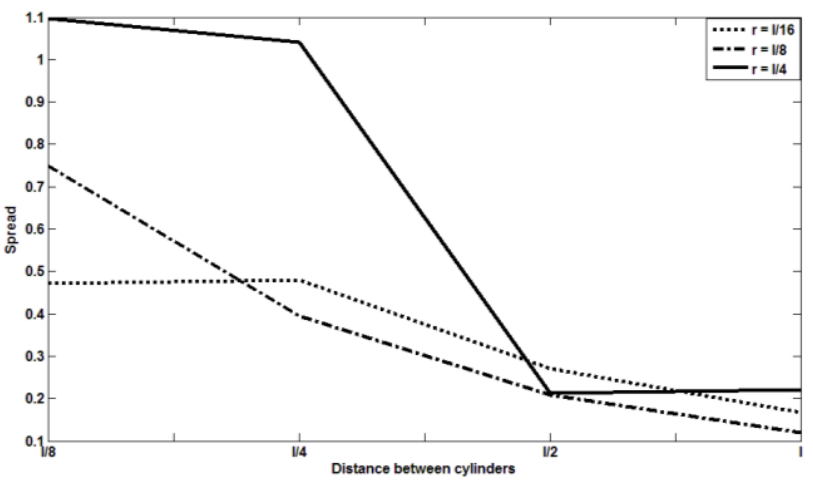

Figure 2.a Mean SAR for the cone division

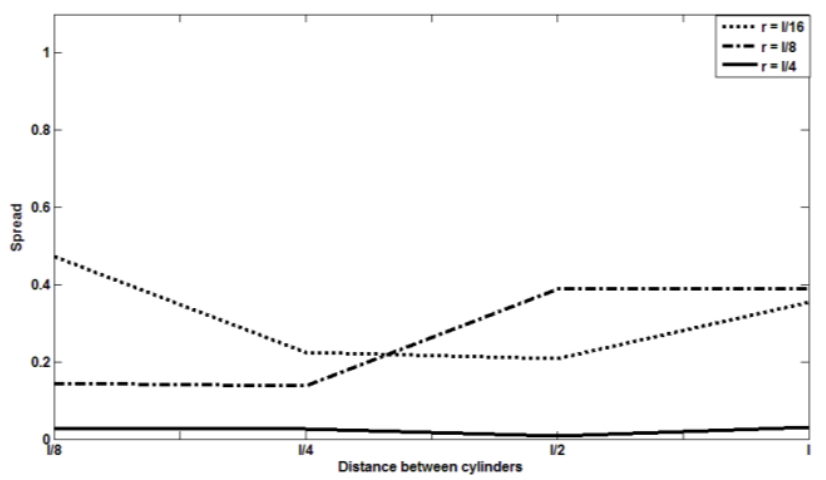

Figure 2.b Mean SAR for the ring division

The results for the cone division, shown in figure 2.a, lead to the conclusion that the radius of the cylinders is the crucial variable. For the three different radii, the spread tends to its minimal value from the distance of $\lambda / 2$ onwards. Thus the angular homogeneity can be reached at this minimal distance between the exposed cylinders.

On the other hand, the results of the concentric rings, figure 2.b, show the distance as the main variable. The largest radius chosen in the simulation presents the best homogeneity independently of the distance. Thus the penetration homogeneity can be reached if the radius is at least greater than $\lambda / 4$. 


\section{Conclusion}

This paper has defined exposition scenario in reverberation chamber of pharmaceutical materials and focus on evaluation of exposure homogeneity. The simulations gives the basic conditions to reach a certain homogeneity of exposure in the case of multi cylinders. These conditions is the minimal radius $(\lambda / 4)$ of the sample and the minimal distance between the samples $(\lambda / 2)$ and are proportional to the used frequency. These first results encourages us to continue in this way. Next steps will we the study of other dispositions and also to realize simulations in three dimensions with a FDTD method.

\section{Acknowledgements}

This work is supported by the Walloon Region as part of the Temptrack project.

\section{References}

1. Uysal I., DeHay W., Altumbas A., Edmond J.-P., Rasmusen R.S. and Ulrich D., Non-thermal effects of Radio Frequency Exposure on biologic pharmaceuticals for RFID applications, IEEE RFID 2010.

2. Liu L., Van Roy S., De Doncker P. and Oestges C., Azimuth radiation pattern characterization of omnidirectional antennas near a human body, in Electromagnetics in Advanced Applications, ICEAA '09, International Conference, Turino, Italia, pp.461-464, Sep. 2009.

3. Jawad O., Lautru D., Benlarbi-Delai A., Dricot J. M., Horlin F. and De Doncker P., A human body model exposed to a cluster of waves: a statistical study of SAR, in Progress In Electromagnetics Research C, Vol 30,1-13,2012 